\title{
1 The Quick and the Dead: Microbial Demography at the Yeast
}

2

4

5 1. Department of Biology, Duke University, Durham, NC 27708, USA

$6 *$ To whom correspondence should be addressed. Email: paul.magwene@duke.edu

$7 \quad$ Dryad data archive DOI: doi:10.5061/dryad.8kn73

8

9

10

11

12

13 Keywords: single-cell analysis, demography, Saccharomyces cerevisiae, heat stress,

14 ecological niche, mitochondrial inheritance 


\section{Abstract}

16 The niche of microorganisms is determined by where their populations can

17 expand. Populations can fail to grow because of high death or low birth rates, but

18 these are challenging to measure in microorganisms. We developed a novel

19 technique that enables single cell measurement of age-structured birth and death

20 rates in the budding yeast, Saccharomyces cerevisiae, and used this method to study

21 responses to heat stress in a genetically diverse panel of strains. We find that

22 individual cells show significant heterogeneity in their rates of birth and death

23 during heat stress. Genotype-by-environment effects on processes that regulate

24 asymmetric cell division contribute to this heterogeneity. These lead to either

25 premature senescence or early life mortality during heat stress, and we find that a

26 mitochondrial inheritance defect explains the early life mortality phenotype of one

27 of the strains we studied. This study demonstrates how the interplay of physiology,

28 genetic variation, and environmental variables influences where microbial

29 populations survive and flourish. 


\section{Introduction}

32 Population growth rate is arguably the most measured variable in all of

33 microbiology. It is used to study the effects of genetic mutations (Breslow et al.

34 2008), as an indicator of physiological stress (Warringer et al. 2003), as a read-out

35 of selection (Vasi, Travisano, and Lenski 1994), and as a proxy for fitness (Joseph

36 and Hall 2004). However, net population growth rate is itself a composite function

37 of the birth and death rates of individuals and measuring only the population

38 growth rate confounds them. Furthermore, birth and death rates frequently change

39 with the age. Because analyses of population growth play such a critical role in

40 studies of microbes, disentangling the effects of birth, death, and aging on

41 population growth should lead to a better understanding of microbial physiology,

42 ecology, and evolution.

43 The range of environmental conditions where the population growth rate of a

44 species is positive corresponds to what the ecologist G. E. Hutchinson called a

45 species' fundamental niche (Hutchinson 1957). The limits of a species' fundamental

46 niche are determined by a combination of physiological, genetic, and demographic

47 factors. One strategy for identifying specific factors that limit the environments

48 where an organism can live is to dissect the demographic mechanisms that drive

49 decreases in population growth rate when a population is exposed to conditions

50 near those limits. Is a decrease in population growth rate driven by a population

51 wide decline in reproductive output as environmental conditions approach their

52 limit? Alternately, are there increases in mortality, perhaps in an age-structured 
53 manner, that limit growth? How do such environmentally induced shifts in

54 demographic parameters vary with genetic background? Questions like these can

55 only be addressed by characterizing the fecundity, mortality, and age of individuals

56 in populations. Such individual level characterization is particularly challenging in

57 microbes due to their small size, large populations, and the consequent challenges of

58 tracking or recovering individual cells.

59 Temperature is an environmental variable that plays a critical role in limiting the

60 fundamental niche of microorganisms. Although the total temperature range over

61 which microbial life is found is quite large (at least $-5^{\circ} \mathrm{C}$ to $113^{\circ} \mathrm{C}$, (Pikuta, Hoover,

62 and Tang 2008)) most species are confined to a relatively modest range of

63 temperatures that support robust growth. The ability to maintain a relatively high

64 growth rate at high temperatures is thought to be critical to the ecological success of

65 the budding yeast Saccharomyces cerevisiae. S. cerevisiae is able to grow at higher

66 temperatures relative to closely related species (Salvadó et al. 2011; Warringer et al.

67 2011). Thermo-tolerance also varies widely between strains of S. cerevisiae (Liti et

68 al. 2009) and has a complex genetic basis (Steinmetz et al. 2002; Sinha et al. 2006).

69 This genetic diversity for growth at high temperatures may also affect human

70 health; a high thermal limit has been hypothesized to contribute to the

71 opportunistic invasion of human hosts by S. cerevisiae (McCusker et al. 1994).

72 Using a newly developed method, called TrackScar, that allows us to

73 simultaneously measure the birth rate, viability, and age of individual cells, we

74 analyzed a genetically diverse panel of yeast strains during growth near their upper 
75 thermal limits. We find that disparate demographic and physiological responses

76 limit population growth of different strains during heat stress. Furthermore, the

77 age-structure of mortality can differ with genetic background, leading to both

78 "premature senescence" as well as "early life mortality." We show that in one

79 genetic background early life mortality likely results from a temperature sensitive

80 mutation affecting mitochondrial inheritance. By measuring the demographic rates

81 of individuals, we elucidate the diverse physiological and demographic factors that

82 limit the niche of budding yeast.

\section{Results}

\section{TrackScar measures the fecundity and mortality of single cells}

85 During the process of asexual mitotic reproduction (budding) in S. cerevisiae and

86 related yeasts, ring-shaped scars of chitin are formed on the cell wall at the site of

87 cytokinesis in both mother and daughter cells. These rings, known as "bud scars",

88 provide a cellular record of the number of daughters an individual cell has

89 produced. Bud scars can be visualized using fluorescently labeled compounds that

90 preferentially bind to chitin (Pringle 1991). Wheat germ agglutinin (WGA), a lectin,

91 is a particularly suitable bud scar stain because it can be can be conjugated to a

92 variety of fluorophores and has low background staining of the cell wall.

93 We developed an experimental method called "TrackScar" for measuring the

94 reproductive output (fecundity) of individual yeast cells based on the sequential

95 staining of bud scars (Fig. 1a,b). The principle behind TrackScar is simple: 1) label a 
96 population of yeast cells at two time points $\left(t_{0}, t_{1}\right)$, using different fluorophores at

97 each time (e.g. cyan at $t_{0}$, yellow at $t_{1}$ ); 2 ) image cells that were stained at both time

98 points; and 3) quantify the number of cell divisions individual cells have undergone

99 in the given time interval by counting the difference in the number of bud scars

100 produced (e.g. no. of yellow scars - no. of cyan scars). The number of daughter cells

101 produced in a given time interval is a discrete and unambiguous measure of the rate

102 of fecundity at the single cell level. Detailed experimental procedures are provided

103 in the supplementary information.

104 Exposing cells to stressful conditions is often accompanied by a decrease in

105 fecundity, with extreme stress leading to cell death. Since two-color TrackScar only

106 measures the number of daughters that a cell produces in one interval, it is possible

107 that cells with low observed fecundity could have died before the first stain (WGA

108 stains both live and dead cells), or in the interval between stains. In order to

109 distinguish between slowly dividing but still viable cells and cells that are dead, the

110 TrackScar method can be extended to include a "recovery phase" (in this case six

111 hours) under permissive growth conditions $\left(30^{\circ} \mathrm{C}\right)$, followed by staining with a third

112 fluorophore. Assuming the recovery phase is sufficiently long, cells that show at

113 least one reproductive event during the recovery phase are still viable; cells that

114 show no growth during recovery are either dead or severely growth arrested (Fig.

115 1c,d). Three-color TrackScar provides more information about individual cells, but

116 data collection is more difficult because mother cells are further diluted during the

117 recovery phase and because more bud scars must be counted. 
118 We performed control experiments to demonstrate that: 1) TrackScar is

119 sensitive; 2) that staining cells using WGA does not affect their division rate; 3) and

120 that six hours of recovery is sufficient to distinguish live from dead cells. These

121 results are described in the Supplementary Information.

\section{Identifying diverse responses to heat stress}

123 The fundamental niche of a species is limited by the range of temperatures

124 ('thermal limits') in which it can survive. Within a species the thermal limit can vary

125 substantially, reflecting genetic diversity. To disentangle the demographic basis of

126 heat sensitivity we applied TrackScar to a genetically diverse panel of S. cerevisiae

127 strains growing in a range of thermal regimes.

128 Identifying slow growing yeast populations

129 We estimated the maximum population growth rates for a panel of 93 sequenced

130 yeast strains (Strope et al. 2015) across a range of temperatures from $21^{\circ} \mathrm{C}$ to $40^{\circ} \mathrm{C}$

131 by measuring the time-varying optical density of liquid cultures grown in a

132 spectrophotometer (Fig. S3). At low temperatures, we observed only small

133 differences between genetic backgrounds in their population growth rates. However

134 at $35.5^{\circ} \mathrm{C}$ and above there is substantial strain-to-strain variation.

135 To identify heat sensitive versus robust strains, we calculated the ratio of

136 maximum growth rates at $35.5^{\circ} \mathrm{C}$, a potentially stressful temperature with high

137 between-strain variance, relative to $30^{\circ} \mathrm{C}$, a standard permissive temperature.

138 Strains with a thermal growth ratio near 1.0 were considered robust to thermal 
139 stress, while those with ratios significantly lower than 1.0 were classified as heat

140 sensitive (Fig. 2a).

141 TrackScar provides a richer view of how population fecundity changes across

142 environments than measuring only the population growth rate. For example, Figure

$1432 \mathrm{~b}$ illustrates how the population distributions of fecundity change over a

144 temperature range of $30^{\circ} \mathrm{C}$ to $40^{\circ} \mathrm{C}$ for diploid S288c cells. S288c grows well at both

$14530^{\circ} \mathrm{C}$ and $35.5^{\circ} \mathrm{C}$. By contrast, at higher temperatures the distribution of fecundities

146 shifts left and there is a noticeable increase in the variance.

\section{Heat sensitivity is associated with the emergence of heterogeneous}

\section{8 subpopulations}

149 We examined the distributions of fecundity for strains that were both sensitive

150 and robust to heat stress. At $30^{\circ} \mathrm{C}$ most strains exhibit unimodal and approximately

151 symmetric fecundity distributions (Fig. S4), although the mean and variances of

152 fecundity differ between backgrounds. At $35.5^{\circ} \mathrm{C}$ robust strains continue to exhibit

153 symmetrical fecundity distributions, with only modest differences in mean

154 fecundity. By contrast, many sensitive strains (Fig. 3; Fig. S4) have asymmetric

155 fecundity distributions, with a substantial increase in slow growing or non-growing

156 cells, even leading to bimodal distributions (Fig. 3; Fig. S4). 


\section{Increased mortality explains slow population growth for some heat}

\section{8 sensitive strains}

159 One mechanism that could lead to asymmetric and bimodal fecundity

160 distributions we observe during heat stress is if some cells died before or during the

161 interval in which we observe their fecundity. To explore this possibility, we used the

162 three-color TrackScar assay to test whether cells with low fecundity during heat

163 stress were viable when the stress is removed. We focused our analyses on three

164 strains - S288C, YJM693, and YJM996 - that have distinct fecundity distributions

165 near their thermal maxima (Fig. 2b, Fig. 3). Since S288C does not exhibit a growth

166 rate decrease at $35.5^{\circ} \mathrm{C}$, we assayed this strain at $40^{\circ} \mathrm{C}$, a temperature where it

167 exhibits a distinct decrease in fecundity and a large number of non-dividing cells.

168 The other two strains were assayed at $35.5^{\circ} \mathrm{C}$. We measured fecundity for six hours

169 during heat stress, incubated the cells overnight at $4^{\circ} \mathrm{C}$, then transferred the cells to

$17030^{\circ} \mathrm{C}$ and measured the number of divisions during a subsequent six-hour period.

171 We used three-color TrackScar to determine whether increased mortality

172 explained the low observed fecundity of cells during heat stress. We compared the

173 number of daughters produced during heat stress of cells that either survived or

174 died during heat stress with the fecundity of unstressed cells. S288C cells with low

175 fecundity during heat stress are frequently alive at the end of the period of stress

176 (Fig. 4). In contrast, viable YJM996 cells frequently produce as many daughters as

177 unstressed cells, but with a broad distribution. Remarkably, and in contrast with the

178 other two strains we examined, the distribution of fecundity among YJM693 cells 
179 that survived heat stress was identical to that of unstressed cells (Kolmogorov-

180 Smirnov test; $\mathrm{p}=0.64$ ). This indicates that heat stress causes YJM693 cells to split

181 into a population of quickly dividing viable cells and another population of unviable

182 cells. Taken together, these results show that in some genetic backgrounds mortality

183 can be the sole cause of slow population growth during heat stress, while in other

184 strains a combination of increased mortality and reduced individual fecundity

185 occurs.

186 Fecundity can be positively or negatively associated with age during stress

187 Yeast mother cells faithfully segregate sub-cellular components to their daughter

188 cells, but also asymmetrically retain aging factors such as damaged proteins and

189 mitochondria (Lai et al. 2002) (McFaline-Figueroa et al. 2011; Aguilaniu et al. 2003).

190 Genetic variation could lead to temperature sensitive mutations that affect either of

191 these processes, which could lead to lower fitness in either younger or older cells,

192 respectively. Since TrackScar works by measuring the replicative age of a cell at the

193 beginning and end of an experiment, the age structure of fecundity emerges

194 naturally during analysis. We will call a group of cells with the same replicative age

195 at the beginning of the experiment a "cohort." To gather sufficient data about older

196 cohorts, we sought out and imaged old cells rather than imaging random cells (see

197 methods).

198 We measured the average fecundity of cohorts of cells in the strains YJM693,

199 YJM996, and S288C. To test whether fecundity was affected by age, we excluded the

200 fecundity of daughter cells because the fecundity of daughters is lower than mothers 
201 due to an extended G1 phase of the cell cycle (Hartwell and Unger 1977)(Fig. S1b).

202 At $30^{\circ} \mathrm{C}$, the average fecundity of cohorts of YJM693 increased slightly with age,

203 whereas the fecundity of cohorts of YJM996 was not significantly affected by age

204 (Fig. 5a)(linear model, $p=0.016$ and $p=0.68$, respectively). The average fecundity

205 of a cohort of S288C was not affected by its age at any temperature (Fig. S5). In

206 contrast, at $35.5^{\circ} \mathrm{C}$, replicative age significantly affects the average fecundity of a

207 cohort in the strains YJM693 and YJM996 (linear model, $p=0.0006$ and $p=0.0008$,

208 respectively). Interestingly, while YJM693 cells produce an average of $0.21 \pm 0.12$

209 fewer daughters in six hours per cohort when heat stressed, YJM996 cells produce

210 an average of $0.33 \pm 0.17$ more daughters in six hours per cohort (intervals are $95 \%$

211 confidence intervals of the mean)(Fig. 5a).

\section{Heat stress can cause premature senescence or early life mortality}

213 The fecundity of YJM693 cells is lower in older cells (Fig. 5a). YJM693 cells

214 that divided less than four times during a six-hour period of heat stress were dead

215 by the end of the experiment (Fig. 4). Consistent with this, we found that the older a

216 cohort of cells was, the more cells in that cohort failed to divide at least four times in

217 six hours-indicating they had died (Fig. 5b). Since, by definition, each cell in a cohort

218 had survived at least long enough to enter the cohort, this indicates that the

219 likelihood of death increases with replicative age at $35.5^{\circ} \mathrm{C}$ in YJM693 and cannot be

220 explained by a model of constant survival risk. Using logistic regression, we estimate

221 that there is a $21 \%(95 \% \mathrm{CI} \pm 6 \%)$ increase in the probability of death for each

222 additional unit of replicative age in this strain during heat stress. These results 
223 suggest that thermal stress affects an asymmetrically inherited aging factor in this

224 genetic background.

225 In contrast to our findings for YJM693, the fecundity of YJM996 cells is higher in

226 older cells (Fig. 5a). Although not all cells with low fecundity in YJM996 died during

227 the exposure to stress, cells that produced no daughters during the six hours of

228 growth at $35.5^{\circ} \mathrm{C}$ were viable less than $5 \%$ of the time (Fig. 4). Therefore, we

229 considered these cells dead for the purpose of estimating age-structured mortality.

230 Younger cohorts in the YJM996 genetic background have substantially higher

231 mortality than older cohorts. More than $80 \%$ of the youngest cells did not produce a

232 daughter cell during 6 hours of recovery at $30^{\circ} \mathrm{C}$, which strongly suggests that they

233 had died (Fig. 5b). Cohorts that had divided only one or two times also had

234 substantially elevated mortality. In contrast, cells in cohorts six and above showed

235 substantially reduced mortality (Fig. 5b). YJM996 thus exhibits an early life

236 mortality phenotype during heat stress. Interestingly, the high mortality of young

237 cells leads older cells to accumulate in the population (Fig. S6). This pattern of

238 mortality in young cells in consistent with heat stress impacting the segregation of a

239 critical cellular component.

\section{Early life mortality is associated with a failure to inherit mitochondria}

242 The early life mortality phenotype of YJM996 during heat stress suggests that this

243 strain may have a temperature-sensitive defect in the segregation of some cellular 
244 component to daughter cells. Previous studies have shown that failure to properly

245 segregate mitochondria (e.g. in an $\Delta m m r 1$ strain) leads to sub-populations of short-

246 and long-lived cells (McFaline-Figueroa et al. 2011), which could generate mortality

247 patterns similar to the ones in YJM996. We examined the morphology of

248 mitochondria at both $30^{\circ} \mathrm{C}$ and $35.5^{\circ} \mathrm{C}$ in strains YJM693, YJM996, and S288C using a

249 mitochondrially localized GFP expressed from a low copy number plasmid. Since

250 this plasmid was uracil selectable, we used $\Delta u r a 3$ derivatives of these strains and

251 grew them in synthetic media lacking uracil.

252 The strain YJM996 has a temperature sensitive defect in mitochondrial

253 morphology and inheritance. In S288C and YJM693, at both $30^{\circ} \mathrm{C}$ and $35.5^{\circ} \mathrm{C}$, the

254 mitochondria form a branching, thread-like network. In contrast, the mitochondria

255 of YJM996 grown at $35.5^{\circ} \mathrm{C}$ formed large globular clumps (Fig. 6). This globular

256 morphology was also present with low ( 10\%) penetrance at $30^{\circ} \mathrm{C}$ (Fig. S7). We

257 noticed that the buds of YJM996 cells often lacked mitochondria even near the end

258 of mitosis (Fig. 6), suggesting that the mitochondria of this strain may be inherited

259 inefficiently. Consistent with this, at $30^{\circ} \mathrm{C}$ and $35.5^{\circ} \mathrm{C}$, approximately $10 \%$ and $75 \%$,

260 respectively, of YJM996 cells lack mitochondria entirely (Fig. S7). We observed cells

261 lacking mitochondria to contain nuclei (Fig. 6) and vacuoles, suggesting that the

262 inheritance of other organelles is not affected. Since mitochondria cannot be created

263 de novo and must be inherited from mother cells, this indicates that mitochondrial

264 inheritance is inhibited by high temperatures in YJM996. 
265 We hypothesized that this mitochondrial inheritance defect was the cause of the

266 early life mortality phenotype in YJM996. Consistent with this, we found that

267 YJM996 cells that lack mitochondria divide fewer times than cells that contain

268 mitochondria at both $30^{\circ} \mathrm{C}$ and $35.5^{\circ} \mathrm{C}$ (Fig. S8). Globular mitochondria were found

269 with low frequency at $30^{\circ} \mathrm{C}$, and we hypothesized that these mitochondria would be

270 associated with slower growth. However, cells with globular mitochondria produced

271 the same number of daughters as cells with wild-type mitochondria, whereas cells

272 without mitochondria produced few or no daughters at either temperature (Fig. S8).

273 This indicates that mortality in this strain is not caused by a defect in mitochondrial

274 morphology per se, although the altered morphology of the mitochondria could

275 contribute to the defect in inheritance. Taken together, these results strongly

276 suggest that YJM693 grows slowly at elevated temperatures due to a defect in the

277 segregation of mitochondria, leading to high mortality in young cells.

\section{Discussion}

279 We have dissected the demographic mechanisms that delimit the fundamental

280 niche of genetically diverse $S$. cerevisiae strains. The growth rate of a population of

281 microbes in the laboratory at a particular temperature can accurately predict the

282 actual environments (the niche) in which a microbe can thrive (Madigan et al.

283 2015). However, the growth rate is the sum of the rates of birth and death, and the

284 effect of temperature on growth rate could be due to a change in either. We

285 developed TrackScar to measure age-structured rates of birth and death in 
286 populations of budding yeast without the need for trapping or microscopic tracking

287 of cells. TrackScar simplifies experimental design and opens the door to studies of

288 microbial demography in environments inaccessible to microscopic tracking of

289 individuals.

290 Single-celled organisms rarely behave identically, even if they share the same

291 genome and the same environment. Random events like stochastic gene expression

292 can cause such differences (Spudich and Koshland 1976; Maamar, Raj, and Dubnau

293 2007; Bumgarner et al. 2012). However, predictable events such as cell cycle and

294 aging (Avery 2006; Levy, Ziv, and Siegal 2012), and epigenetic inheritance of growth

295 rate (Ziv, Siegal, and Gresham 2013; Levy, Ziv, and Siegal 2012) can also contribute.

296 Genetic variation can impact cellular individuality, indicating that it could be

297 selected for during evolution (Ziv, Siegal, and Gresham 2013; Fehrmann, Bottin-

298 Duplus, et al. 2013; Holland et al. 2013). This individuality makes the average a poor

299 predictor of the behavior of individuals, and can obscure the physiological basis of

300 population-level phenotypes.

301 Heat stress often causes extensive variability in the fitness of individual cells,

302 as shown by the asymmetric and frequently bimodal distributions of the fecundity

303 we observed. This variability can be age structured, and in at least one case is a

304 consequence of the failure of cells to appropriately segregate sub-cellular

305 components (see below). The ability to easily distinguish mother and daughter cells

306 in budding yeast make these patterns particularly apparent, but subtle asymmetries

307 also lead to aging in ostensibly symmetrically dividing organisms such as 
308 Escherichia coli (Stewart et al. 2005). We speculate that genetic variation in cellular

309 mechanisms that establish or normalize asymmetries between mother and daughter

310 cells may be an important cause of phenotypic variability in other organisms.

311 Active processes ensure that sufficient quantities of organelles and other

312 sub-cellular components are segregated to daughter cells (Chernyakov, Santiago-

313 Tirado, and Bretscher 2013; Marston 2014) while at the same time damaged

314 proteins, organelles and other aging factors are asymmetrically retained in the

315 mother cell, eventually leading to senescence (Lai et al. 2002; McFaline-Figueroa et

316 al. 2011; Aguilaniu et al. 2003; Sinclair and Guarente 1997; Dillin, Gottschling, and

317 Nyström 2014). Mutations affecting these two processes could lead to different

318 demographic outcomes. Aberrant production or distribution of an asymmetrically

319 distributed factor could lead to premature aging, whereas the failure to produce or

320 distribute a critical sub-cellular component could lead to newly born cells dying.

321 We found that fecundity and mortality were age-structured in two clinical

322 isolates. In the strain YJM693, older cohorts showed lower fecundity and higher

323 mortality, indicating that YJM693 cells senesce prematurely at elevated

324 temperatures. Whatever causes senescence in budding yeast must be

325 asymmetrically retained in mother cells (Kennedy, Austriaco, and Guarente 1994). A

326 number of molecular mechanisms are associated with senescence in good

327 conditions in yeast including autonomously replicating circularized rDNA, damaged

328 vacuoles and mitochondria, and the aggregation of damaged proteins (Lai et al.

329 2002; McFaline-Figueroa et al. 2011; Aguilaniu et al. 2003; Sinclair and Guarente 
330 1997; Dillin, Gottschling, and Nyström 2014), although some of these remain

331 controversial (Fehrmann, Paoletti, et al. 2013). We speculate that the strain YJM693

332 contains a temperature sensitive mutation in a gene required for normal replicative

333 lifespan.

334 In contrast to the strain YJM693, older cells in the strain YJM996 were more

335 fecund than younger cells. The low fecundity of young cohorts in these strains is

336 partly, but not entirely, due to very high mortality in young cohorts. In contrast, the

337 mortality of older cohorts is low, leading them to accumulate in the population. The

338 force of natural selection on fecundity of a cohort scales with the relative

339 survivorship of that cohort (Hamilton 1966). This implies that natural selection on

340 the fecundity of older cells is much stronger in this strain, but only during heat

341 stress.

342 The early life mortality of YJM996 is linked to a defect in mitochondrial

343 inheritance. YJM996 cells contain mitochondria with an aberrant globular

344 morphology during heat stress, and daughter cells in this strain frequently fail to

345 inherit mitochondria. Although mtDNA is dispensable in S. cerevisiae, the

346 mitochondria themselves are essential (Kispal et al. 2005; Chernyakov, Santiago-

347 Tirado, and Bretscher 2013; Frederick, Okamoto, and Shaw 2008). This strongly

348 suggests that the failure to inherit mitochondria causes the high mortality in young

349 cells in this strain, although we cannot rule out that other essential components may

350 fail to be segregated as well. 
351 Genetic variation affects the relative contribution of mortality and fecundity to

352 stress-induced changes population growth rate. For example, increased mortality

353 was both necessary and sufficient to explain the slow growth of the clinical isolate

354 YJM693. Cells that survived chronic heat stress in this strain had fecundity identical

355 to unstressed cells, whereas nearly all cells with low fecundity were dead. In

356 contrast, cells with low fecundity during heat stress in the clinical isolate YJM996 or

357 the genomic reference strain S288C are sometimes alive. Our results indicate that

358 changes in population growth rate should be interpreted cautiously, since they can

359 reflect the effects of distinct demographic mechanisms.

360 The ability to grow at high temperatures in the presence of alcohol is a key

361 innovation critical to the ecological success of Saccharomyces cerevisiae (Goddard

362 2008). In this study, we examined the standing genetic variation present within $S$.

363 cerevisiae for the ability to grow at high temperatures. The ability of cells to grow at

364 high temperatures has been hypothesized to be a trait that enables pathogenesis in

365 yeast, but we show that clinical isolates can also be sensitive to heat. Thermal

366 tolerance in yeast is a genetically complex trait (Steinmetz et al. 2002; Sinha et al.

367 2006), and consistent with this we found that heat can operate on distinct

368 demographic functions to lower growth rate, reflecting effects on different cellular

369 processes involved in asymmetric division in budding yeast. Our results connect

370 physiology to demography, and shed light on how genetic variation affects the

371 thermal limits of budding yeast. 


\section{Methods}

374 All of our experiments were conducted using clonal populations grown in YPD or

375 in SC-ura media when a plasmid was used. Before staining, cells were propagated

376 for at least 16 hours in log-phase growth at the temperature being surveyed. Cells

377 were first stained with Alexa488-conjugated wheat germ agglutinin (WGA) for 15

378 min. Cells were washed once in YPD and diluted to $0.33 \times 10^{6}$ cells $/ \mathrm{ml}$, then grown

379 for six hours. Cells were then fixed in PBS $+8 \%$ formaldehyde, and stored until

380 imaging. Before imaging, cells were stained as above with tetramethylrhodamine

381 (TMR)-conjugated WGA. Widefield Z-stacks were acquired, deconvolved, and then

382 processed using a maximum pixel intensity projection. The numbers of buds stained

383 by each dye were counted by manually from these projections. At least 150 fields of

384 view which contained at least one cell with the first stain were collected for each

385 biological replicate. This number was chosen to keep the acquisition time required

386 for a biological replicate of a two-color experiment below one hour. Growth curves

387 were collected on a Tecan Sunrise over a 48hr period with measurements every 15

388 minutes. See Supplementary Information for detailed methods including for three-

389 color TrackScar.

\section{Acknowledgements}

391 We acknowledge Nicolas Buchler, Daniel Lew, Daniel Skelly, and Helen Murphy

392 for helpful comments on the manuscript. We thank Debra Murray for suggesting the

393 name "TrackScar". This work was supported in part by NIH (P50GM081883) and

394 NSF (MCB1330545) awards to P. M. M. 


\section{Author contributions}

396 C.S.M designed the experiments, carried out the experiments, analyzed data, and

397 prepared the manuscript. P.M.M. designed the experiments, and prepared the

398 manuscript

\section{Competing financial interests}

$400 \quad$ The authors declare no competing financial interests.

\section{Materials and correspondence}

402 Material requests and correspondence should be directed to Paul M. Magwene.

\section{References}

404 Aguilaniu, Hugo, Lena Gustafsson, Michel Rigoulet, and Thomas Nyström. 2003.

405 “Asymmetric Inheritance of Oxidatively Damaged Proteins During Cytokinesis.."

$406 \quad$ Science 299 (5613): 1751-53. doi:10.1126/science.1080418.

407 Avery, Simon V. 2006. "Microbial Cell Individuality and the Underlying Sources of

408 Heterogeneity." Nat Rev Micro 4 (8): 577-87. doi:10.1038/nrmicro1460.

409 Breslow, David K, Dale M Cameron, Sean R Collins, Maya Schuldiner, Jacob Stewart-

410 Ornstein, Heather W Newman, Sigurd Braun, Hiten D Madhani, Nevan J Krogan,

411 and Jonathan S. Weissman. 2008. "A Comprehensive Strategy Enabling High-

412 Resolution Functional Analysis of the Yeast Genome." Nat Meth 5 (8). Nature

413 Publishing Group: 711-18. doi:10.1038/nmeth.1234.

414 Bumgarner, Stacie L., Gregor Neuert, Benjamin F. Voight, Anna Symbor-Nagrabska, 
415 Paula Grisafi, Alexander van Oudenaarden, and Gerald R Fink. 2012. "Single-Cell

416 Analysis Reveals That Noncoding RNAs Contribute to Clonal Heterogeneity by

417 Modulating Transcription Factor Recruitment." Molecular Cell. Cell Press.

418 Chernyakov, Irina, Felipe Santiago-Tirado, and Anthony Bretscher. 2013. “Active

419 Segregation of Yeast Mitochondria by Myo2 Is Essential and Mediated by Mmr1

420 and Ypt11.." Current Biology : CB 23 (18): 1818-24.

421 doi:10.1016/j.cub.2013.07.053.

422 Dillin, Andrew, Daniel E. Gottschling, and Thomas Nyström. 2014. "The Good and the

423 Bad of Being Connected: the Integrons of Aging.." Current Opinion in Cell Biology

424 (February): 107-12. doi:10.1016/j.ceb.2013.12.003.

425 Fehrmann, Steffen, Camille Paoletti, Youlian Goulev, Andrei Ungureanu, Hugo

426 Aguilaniu, and Gilles Charvin. 2013. “Aging Yeast Cells Undergo a Sharp Entry

427 Into Senescence Unrelated to the Loss of Mitochondrial Membrane Potential..”

428 Cell Reports 5 (6): 1589-99. doi:10.1016/j.celrep.2013.11.013.

429 Fehrmann, Steffen, Hélène Bottin-Duplus, Andri Leonidou, Esther Mollereau, Audrey

430 Barthelaix, Wu Wei, Lars M Steinmetz, and Gaël Yvert. 2013. "Natural Sequence

431 Variants of Yeast Environmental Sensors Confer Cell-to-Cell Expression

432 Variability.." Molecular Systems Biology 9: 695. doi:10.1038/msb.2013.53.

433 Frederick, Rebecca L, Koji Okamoto, and Janet M Shaw. 2008. "Multiple Pathways

$434 \quad$ Influence Mitochondrial Inheritance in Budding Yeast.." Genetics 178 (2).

435 Genetics: 825-37. doi:10.1534/genetics.107.083055.

436 Goddard, Matthew R. 2008. “Quantifying the Complexities of Saccharomyces

437 Cerevisiae'S Ecosystem Engineering via Fermentation." Ecology 89 (8). 
438 Ecological Society of America: 2077-82. doi:10.1890/07-2060.1.

439 Hamilton, W D. 1966. “The Moulding of Senescence by Natural Selection.” Journal of

$440 \quad$ Theoretical Biology 12 (1): 12-45.

441 Hartwell, L.H., and M W Unger. 1977. “Unequal Division in Saccharomyces

442 Cerevisiae and Its Implications for the Control of Cell Division..” The Journal of

$443 \quad$ Cell Biology 75 (2 Pt 1): 422-35.

444 Holland, Sara L, Tom Reader, Paul S Dyer, and Simon V. Avery. 2013. "Phenotypic

445 Heterogeneity Is a Selected Trait in Natural Yeast Populations Subject to

446 Environmental Stress.." Environmental Microbiology, August. doi:10.1111/1462-

$447 \quad 2920.12243$.

448 Hutchinson, G E. 1957. “Concluding Remarks.” Cold Spring Harbor Symposia on

449 Quantitative Biology, no. 22. Cold Spring Harbor Symposia on Quantitative

$450 \quad$ Biology: 415-27.

451 Joseph, Sarah B, and David W Hall. 2004. “Spontaneous Mutations in Diploid

452 Saccharomyces Cerevisiae More Beneficial Than Expected." Genetics 168 (4).

453 Genetics Society of America: 1817-25. doi:10.1534/genetics.104.033761.

454 Kennedy, B K, N R Austriaco, and L Guarente. 1994. “Daughter Cells of

455 Saccharomyces Cerevisiae From Old Mothers Display a Reduced Life Span..” The

456 Journal of Cell Biology 127 (6 Pt 2). Rockefeller Univ Press: 1985-93.

457 doi:10.1083/jcb.127.6.1985.

458 Kispal, Gyula, Katalin Sipos, Heike Lange, Zsuzsanna Fekete, Tibor Bedekovics,

459 Tamás Janáky, Jochen Bassler, et al. 2005. “Biogenesis of Cytosolic Ribosomes

460 Requires the Essential Iron-Sulphur Protein Rli1p and Mitochondria." The EMBO 
461 Journal 24 (3). EMBO Press: 589-98. doi:10.1038/sj.emboj.7600541.

462 Lai, Chi-Yung, Ewa Jaruga, Corina Borghouts, and S Michal Jazwinski. 2002. “A

463 Mutation in the ATP2 Gene Abrogates the Age Asymmetry Between Mother and

464 Daughter Cells of the Yeast Saccharomyces Cerevisiae.." Genetics 162 (1): 73-87.

465 Levy, Sasha F., Naomi Ziv, and Mark L. Siegal. 2012. “Bet Hedging in Yeast by

466 Heterogeneous, Age-Correlated Expression of a Stress Protectant." PLoS Biol 10

467 (5). Public Library of Science: e1001325EP-.

468 doi:doi:10.1371/journal.pbio.1001325.

469 Liti, Gianni, David M Carter, Alan M Moses, Jonas Warringer, Leopold Parts, Stephen

470 A James, Robert P Davey, et al. 2009. "Population Genomics of Domestic and

$471 \quad$ Wild Yeasts." Nature 458 (7236).

472 Maamar, Hédia, Arjun Raj, and David Dubnau. 2007. “Noise in Gene Expression

473 Determines Cell Fate in Bacillus Subtilis.." Science 317 (5837). American

474 Association for the Advancement of Science: 526-29.

475 doi:10.1126/science.1140818.

476 Madigan, Michael T, John M Martinko, Kelly S Bender, Daniel H Buckley, and David A

477 Stahl. 2015. Brock Biology of Microorganisms. 14 ed. Vol. 4. Boston: Pearson.

478 Marston, Adele L. 2014. “Chromosome Segregation in Budding Yeast: Sister

479 Chromatid Cohesion and Related Mechanisms..” Genetics 196 (1): 31-63.

480 doi:10.1534/genetics.112.145144.

481 McCusker, J H, K V Clemons, D A Stevens, and R W Davis. 1994. “Genetic

482 Characterization of Pathogenic Saccharomyces Cerevisiae Isolates.." Genetics

$483136(4): 1261-69$. 
484 McFaline-Figueroa, José Ricardo, Jason Vevea, Theresa C Swayne, Chun Zhou,

485 Christopher Liu, Galen Leung, Istvan R Boldogh, and Liza A Pon. 2011.

486 "Mitochondrial Quality Control During Inheritance Is Associated with Lifespan

487 and Mother-Daughter Age Asymmetry in Budding Yeast.." Aging Cell 10 (5).

488 Blackwell Publishing Ltd: 885-95. doi:10.1111/j.1474-9726.2011.00731.x.

489 Pikuta, Elena V, Richard B Hoover, and Jane Tang. 2008. "Microbial Extremophiles at

490 the Limits of Life." Critical Reviews in Microbiology 33 (3). Taylor \& Francis: 183-

491 209. doi:10.1080/10408410701451948.

492 Pringle, J.R. 1991. "Staining of Bud Scars and Other Cell Wall Chitin with Calcofluor..”

$493 \quad$ Methods in Enzymology 194: 732-35.

494 Salvadó, Z, F N Arroyo-López, J M Guillamón, G Salazar, A Querol, and E Barrio. 2011.

495 "Temperature Adaptation Markedly Determines Evolution Within the Genus

496 Saccharomyces.." Applied and Environmental Microbiology 77 (7). American

497 Society for Microbiology: 2292-2302. doi:10.1128/AEM.01861-10.

498 Sinclair, David A, and Leonard Guarente. 1997. "Extrachromosomal rDNA Circles: a

499 Cause of Aging in Yeast." Cell. Cell Press.

500 Sinha, Himanshu, Bradly P Nicholson, Lars M Steinmetz, and John H McCusker. 2006.

501 “Complex Genetic Interactions in a Quantitative Trait Locus.” PLoS Genet 2 (2).

502 Public Library of Science: e13. doi:10.1371/journal.pgen.0020013.

503 Spudich, J L, and D E Koshland. 1976. "Non-Genetic Individuality: Chance in the

504 Single Cell.." Nature 262 (5568): 467-71.

505 Steinmetz, Lars M, Himanshu Sinha, Dan R Richards, Jamie I Spiegelman, Peter J

506 Oefner, John H McCusker, and Ronald W. Davis. 2002. “Dissecting the 

30. doi:10.1038/416326a.

509 Stewart, Eric J., Richard Madden, Gregory Paul, and François Taddei. 2005. “Aging

510 and Death in an Organism That Reproduces by Morphologically Symmetric

511 Division." Edited by Thomas Kirkwood. PLoS Biol 3 (2). Public Library of

512 Science: e45. doi:10.1371/journal.pbio.0030045.

513 Strope, Pooja K, Daniel A. Skelly, Stanislav G Kozmin, Gayathri Mahadevan, Eric A

514 Stone, Paul M. Magwene, Fred S Dietrich, and John H McCusker. 2015. “The 100-

515 Genomes Strains, an S. Cerevisiae Resource That Illuminates Its Natural

516 Phenotypic and Genotypic Variation and Emergence as an Opportunistic

517 Pathogen.." Genome Research 25 (5). Cold Spring Harbor Lab: 762-74.

518 doi:10.1101/gr.185538.114.

519 Vasi, Farida, Michael Travisano, and Richard E Lenski. 1994. “Long-Term

520 Experimental Evolution in Escherichia Coli. II. Changes in Life-History Traits

521 During Adaptation to a Seasonal Environment." The American Naturalist 144 (3).

522 The University of Chicago Press for The American Society of Naturalists: 432-

$523 \quad 56$. doi: $10.2307 / 2462954$.

524 Warringer, Jonas, Elke Ericson, Luciano Fernandez, Olle Nerman, and Anders

525 Blomberg. 2003. "High-Resolution Yeast Phenomics Resolves Different

526 Physiological Features in the Saline Response." Proc Natl Acad Sci USA 100 (26):

$527 \quad 15724-29$.

528 Warringer, Jonas, Enikö Zörgö, Francisco A Cubillos, Amin Zia, Arne Gjuvsland, Jared

529 T Simpson, Annabelle Forsmark, et al. 2011. "Trait Variation in Yeast Is Defined 
$530 \quad$ by Population History.." PLoS Genet 7 (6). Public Library of Science: e1002111.

531 doi:10.1371/journal.pgen.1002111.

532 Ziv, Naomi, Mark L. Siegal, and David Gresham. 2013. “Genetic and Non-Genetic

533 Determinants of Cell-Growth Variation Assessed by High-Throughput

$534 \quad$ Microscopy.." Molecular Biology and Evolution, August.

535 doi:10.1093/molbev/mst138.

536

537 


\section{Figures}

A

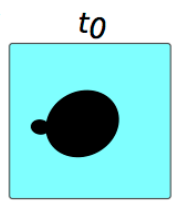

C

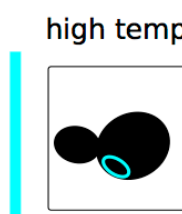

growth

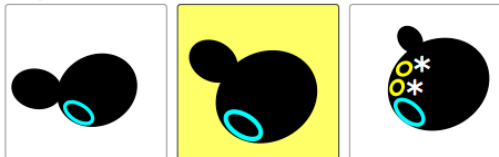

p.

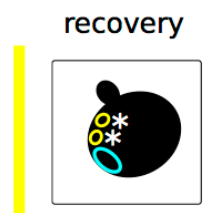

live

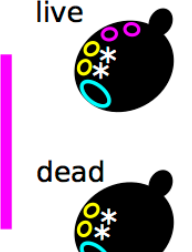

B
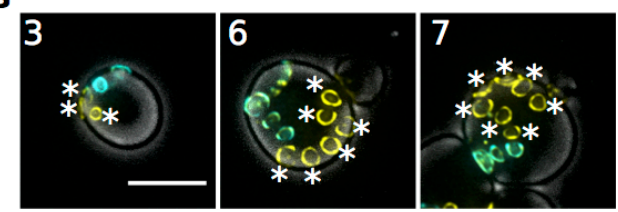

D

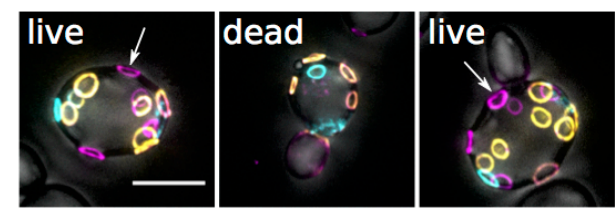

540 Figure 1. TrackScar measures the fecundity and viability of individual yeast. A) A

541 schematic of two-color TrackScar is shown. To measure the reproductive output of

542 individuals, cells are: 1) stained with wheat germ agglutinin (WGA) conjugated to

543 Alexa488 (cyan) at $t_{0} ; 2$ ) grown in the absence of the stain; 3 ) stained with WGA

544 conjugated to tetramethylrhodamine (yellow) at $t_{1}$. New buds (labeled with

545 asterixes) are only stained by the second dye. B) Examples of haploid S288C cells

546 that produced different numbers of daughters during $6 \mathrm{hr}$ of growth at $30^{\circ} \mathrm{C}$ in YPD

547 are shown. C) A schematic of three-color TrackScar is shown. Two colors of WGA

548 (cyan and yellow) are used to measure the fecundity of cells during heat stress. To

549 determine viability, the cells are transferred to permissive conditions and then

550 stained with a third color of WGA (magenta). Viable cells produce new buds that are

551 only stained by the third dye. D) Examples of diploid YJM693 cells that were either

552 viable or inviable after exposure to growth at $35.5^{\circ} \mathrm{C}$ are shown. Arrows highlight

553 one of the bud scars produced by live cells in permissive conditions. All micrographs

554 are maximum intensity projections of z-stacks. Scale bar shows five microns. 


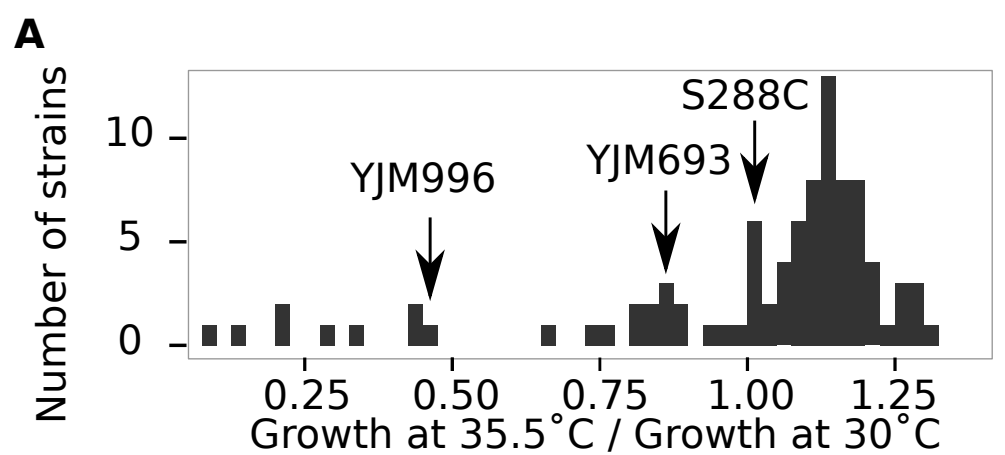

555

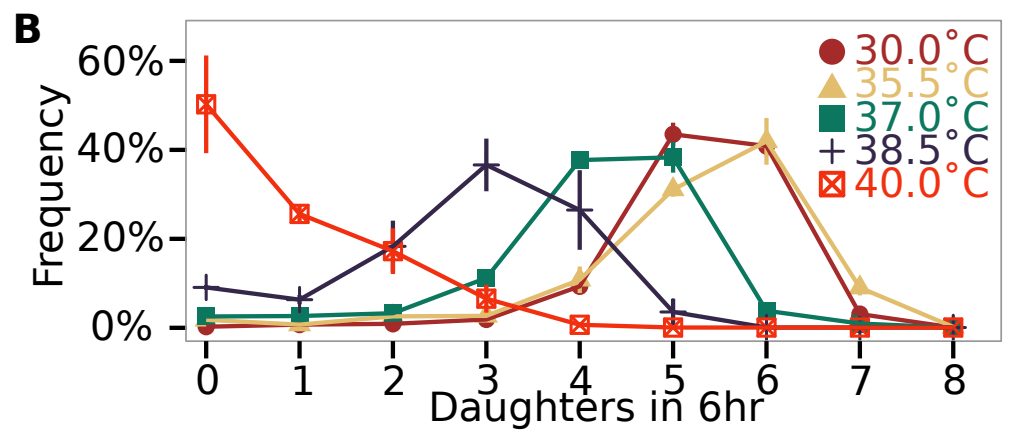

556 Figure 2. There is extensive genetic diversity in thermotolerance among

557 environmental yeast isolates. A) A histogram of the growth rate of each of 93 yeast

558 strains at $35.5^{\circ} \mathrm{C}$ normalized to their growth rate at $30^{\circ} \mathrm{C}$ is shown. B) Frequency

559 polygons of the number of daughters produced by cells in the strain S288C during 6

$560 \mathrm{hr}$ of growth at $30^{\circ} \mathrm{C}$ (brown circles), $35.5^{\circ} \mathrm{C}$ (gold triangles), $37^{\circ} \mathrm{C}$ (green squares),

$56138.5^{\circ} \mathrm{C}$ (black crosses), and $40^{\circ} \mathrm{C}$ (red crossed boxes) are shown. Error bars are the

562 standard error of the mean for three biological replicates. 


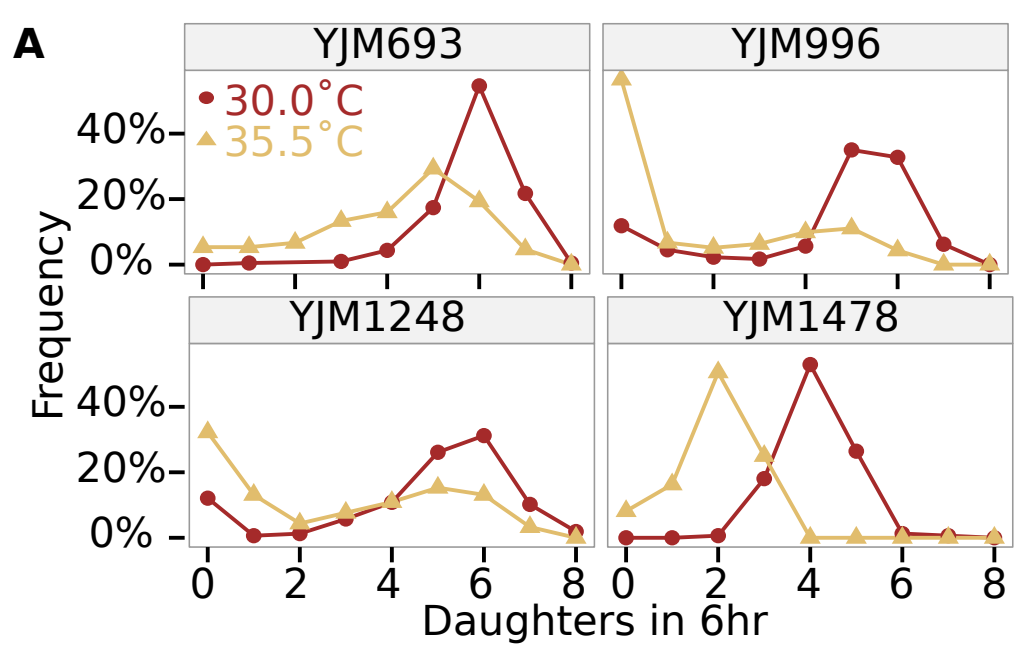

565 Figure 3. Strains that are sensitive to heat stress frequently have subpopulations

566 with very low fecundity. Plots showing frequency polygons of the number of

567 daughters produced in $6 \mathrm{hr}$ at $30^{\circ} \mathrm{C}$ (brown circles) and $35.5^{\circ} \mathrm{C}$ (gold triangles) are

568 shown for $\mathbf{A}$ ) robust and B) sensitive strains are shown. A single representative

569 replicate is shown for each temperature for each strain.

570 


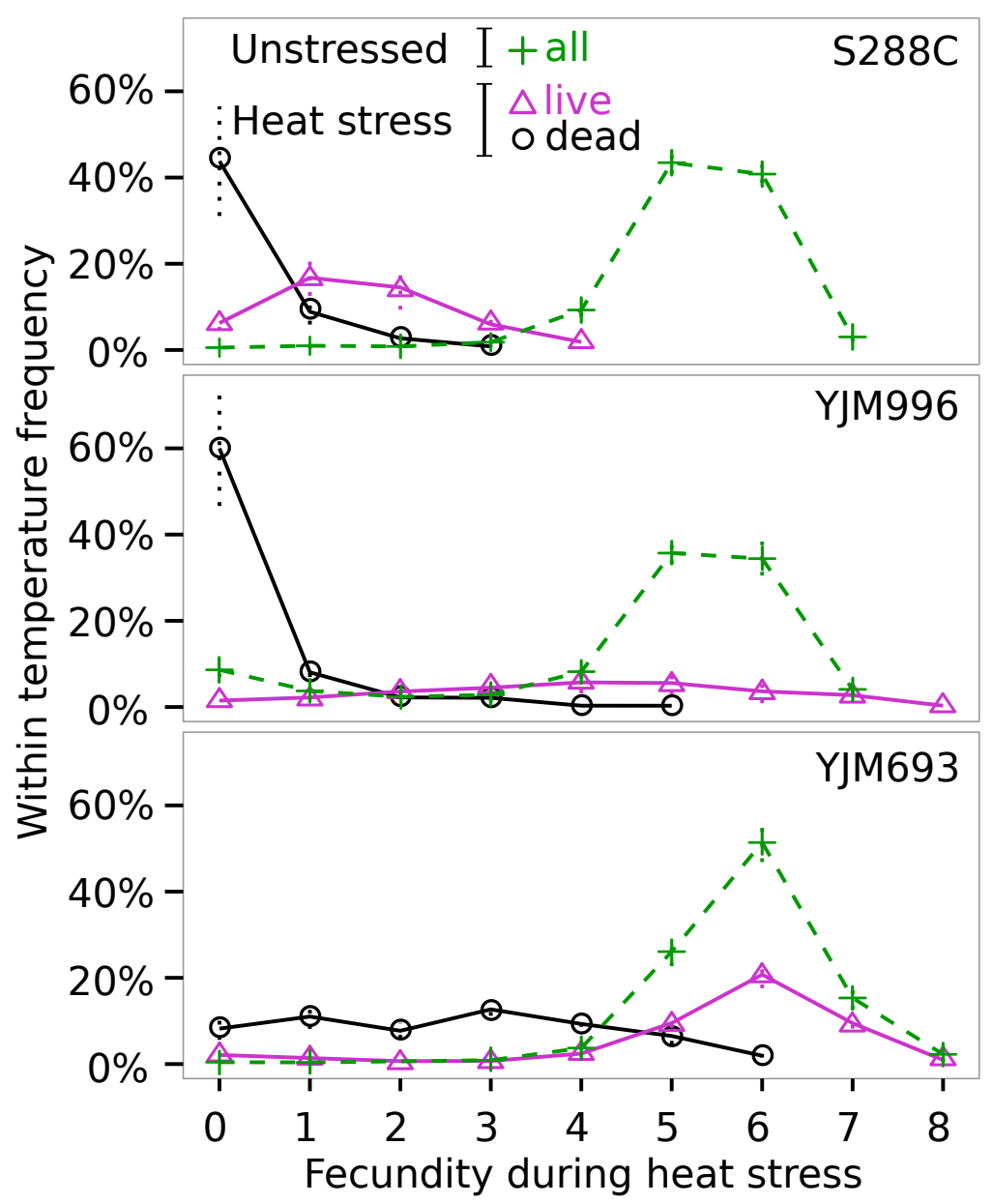

572 Figure 4. Mortality contributes to slow population growth in a genotype specific

573 manner. Cells were heat stressed at either $35.5^{\circ} \mathrm{C}$ or $40^{\circ} \mathrm{C}$, then assayed for viability

574 at $30^{\circ} \mathrm{C}$. The frequency polygons plot the fecundity during heat stress for cells that

575 were subsequently classified as viable (magenta triangles) and inviable (black

576 circles) from strains S288C, YJM996, and YJM693. The fecundity of unstressed cells

577 is plotted as a dashed line with green crosses. Error bars are the standard error of

578 the mean for three biological replicates. 

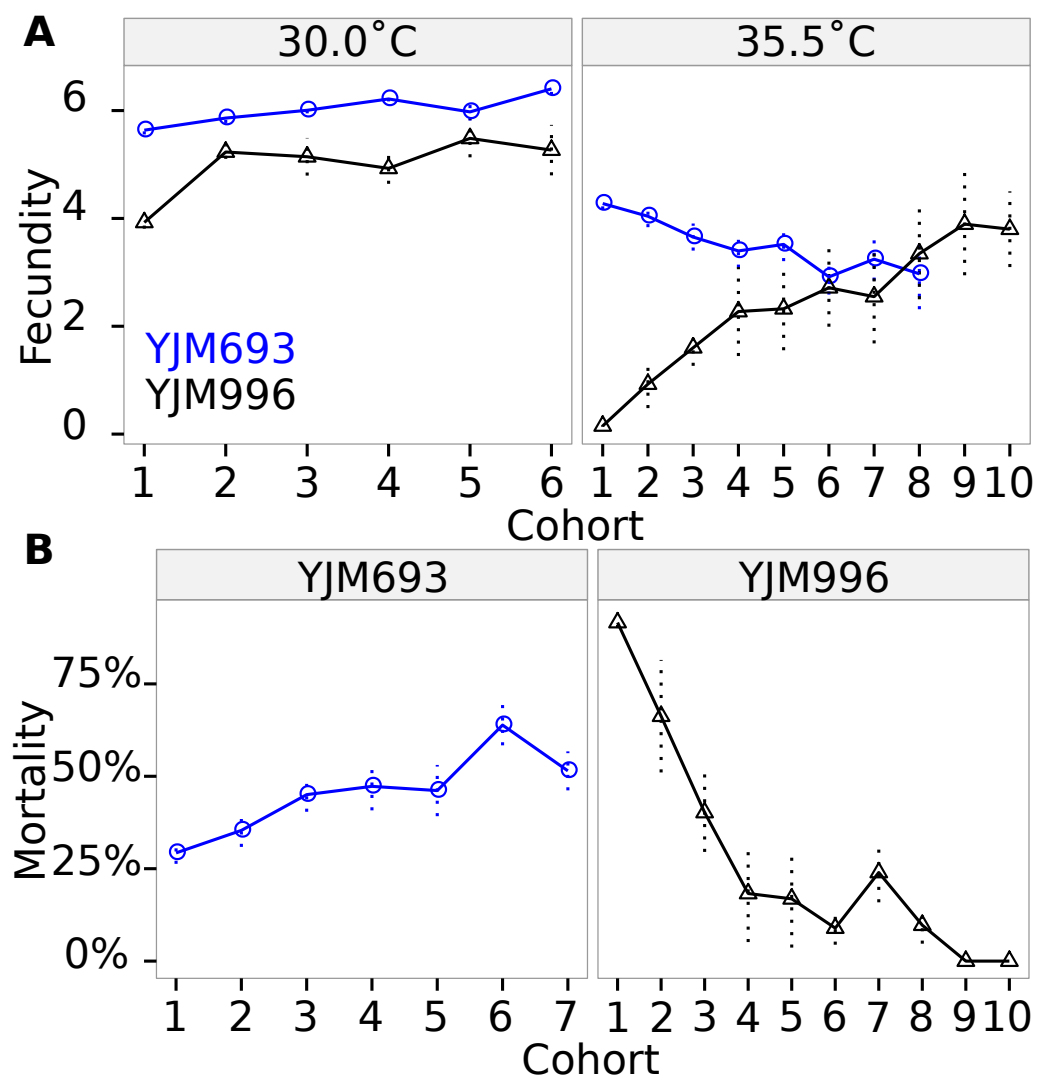

580 Figure 5. Genetic background determines the age structure of fecundity and

581 mortality during heat stress. A) The average fecundity of a cohort during 6hr growth

582 at either $30^{\circ} \mathrm{C}$ or $35.5^{\circ} \mathrm{C}$ for the strains YJM693 (blue circles) and YJM996 (black

583 triangles). Error bars are the standard error of the mean for at least three biological

584 replicates. Only cohorts with at least three cells in each of the three biological

585 replicates are shown. B) The mortality of cohorts of YJM693 and YJM996 cells is

586 shown. YJM693 cells were considered "dead" if they divided four times or fewer

587 growth at $35.5^{\circ} \mathrm{C}$, whereas YJM996 cells were considered "dead" if they did not

588 divide at all during six hours of growth at $35.5^{\circ} \mathrm{C}$. Error bars show the standard error

589 of the mean for at least three biological replicates. Only cohorts with at least five

590 cells in each of the three biological replicates are shown. 


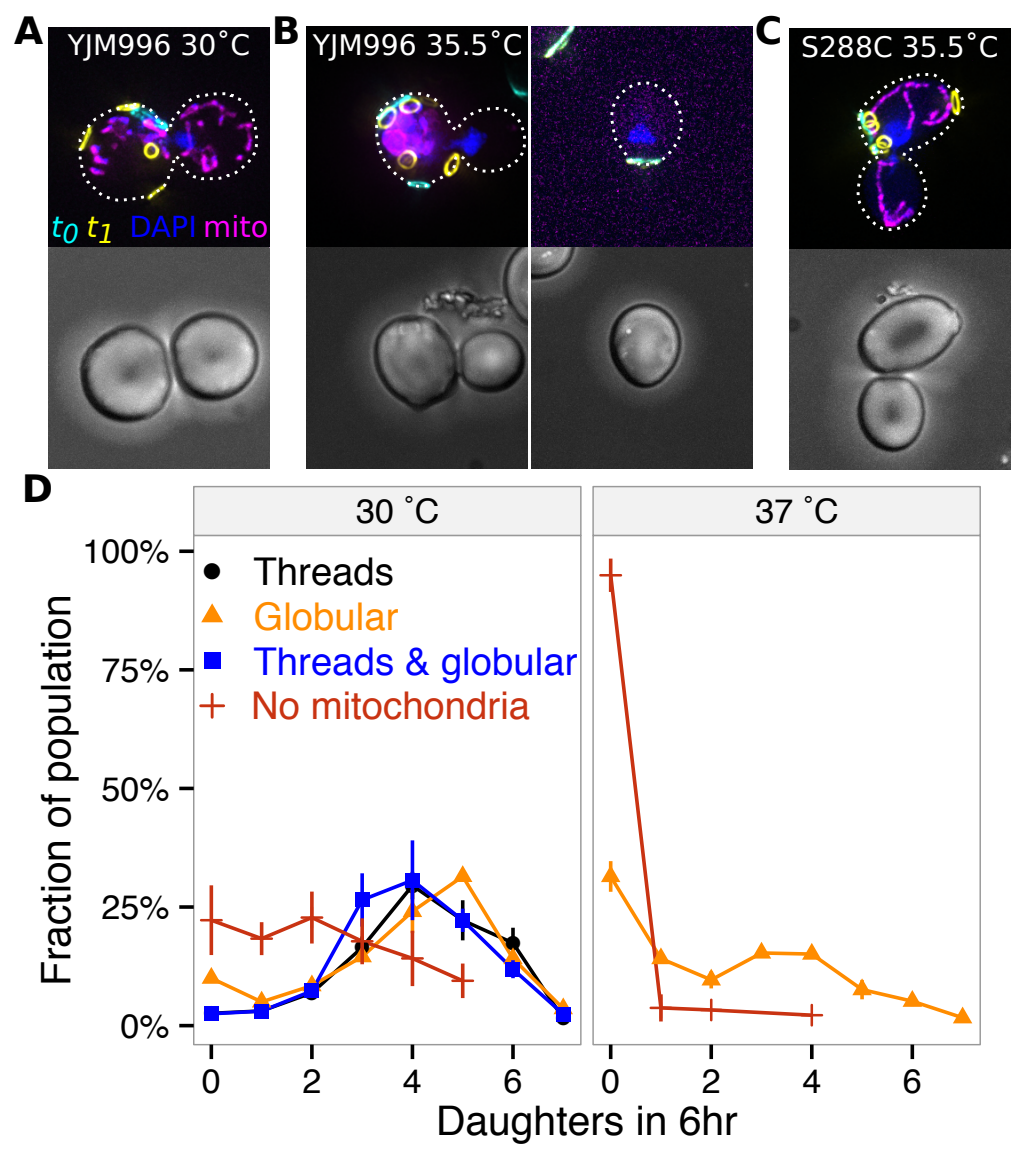

592 Figure 6. Early life mortality is associated with a defect in mitochondrial

593 inheritance in the YJM996 genetic background. Representative images are shown of

594 TrackScar staining at zero hours (cyan) and six hours (yellow), DAPI staining (blue),

595 and mitochondria-localized GFP (magenta) for a $\Delta$ ura3 derivative of the strain

596 YJM996 at A) $30^{\circ} \mathrm{C}$ and B) $35.5^{\circ} \mathrm{C}$, and a $\Delta$ ura3 S288C derivative at C) $35.5^{\circ} \mathrm{C}$. Each

597 budding cell is staged in the process of nuclear division to facilitate comparison of

598 mitochondrial inheritance. Dotted lines show the outlines of the cells. D) The

599 fecundity of cells with thread-like mitochondrial morphology, globular morphology,

600 a mixture of clumps and threads, and cells that lack mitochondria is shown for cells

601 in the YJM996 genetic background at both $30^{\circ} \mathrm{C}$ and $35.5^{\circ} \mathrm{C}$. Error bars show the

602 standard error of the mean for three biological replicates of at least 150 cells. 


\section{Supplementary information for:}

2 The Quick and the Dead: Microbial Demography at the Yeast Thermal Limit

1. Department of Biology, Duke Center for Systems Biology, Duke University, Durham, NC 27708, USA

$8 *$ To whom correspondence should be addressed. Email: paul.magwene@duke.edu 


\section{Supplementary Information Table of Contents}

\section{Supplementary Introduction}

12 Supplementary Results

13 TrackScar provides a sensitive measure for differences in fecundity

$14 \quad$ TrackScar minimally affects cellular physiology

15 Population growth rate and mean fecundity are well-correlated

16 Six hours of recovery is sufficient to distinguish live from dead cells

17 Heat stress can alter the distribution of ages in a population

\section{Supplementary Methods}

$19 \quad$ TrackScar Staining

20

Microscopy

Image Processing and Data Analysis

Data Collection and Microscopy for Time Series

Growth Curves

\section{Supplementary References}

\section{Supplementary Figures}

Figure S1. TrackScar is sensitive and minimally affects cellular physiology 
Figure S3. Growth rate of all strains as a function of temperature

Figure S4. Histograms of all strains analyzed using TrackScar

Figure S5. Age-structured fecundity of S288C

$34 \quad$ Figure S8 Fecundity of cells with globular mitochondria

\section{Supplementary Tables}




\section{Supplementary Results}

\section{TrackScar minimally affects cellular physiology}

40 A potential concern with any vital-staining method is the possibility that it

41 could change the cell physiological processes of interest. To explore this possibility,

42 we compared division time estimates based on TrackScar to results from previous

43 studies using timelapse microscopy. Using TrackScar we estimated the average

44 division time to be 73.9 minutes for haploid cells of the genomic reference strain

45 S288c grown in rich-media conditions. This is very similar to an estimate of 72.6

46 minutes derived previously for this same strain using timelapse microscopy (Lord

47 and Wheals 1981). To further explore the physiological impact of WGA staining on

48 cellular division rates, we carried out a TrackScar experiment in which the time

49 interval between the first and second stain was varied between one and six hours.

50 We reasoned that if WGA staining is a significant cellular stress that the

51 reproductive rates immediately following the first stain should be systematically

52 lower than in later time points, because extended incubation would allow cells to

53 recover from any physiological perturbations caused by staining. We carried out

54 this experiment for nine genetically diverse $S$. cerevisiae strains. We found no

55 evidence that reproductive rates at earlier time points were any lower than later

56 time points (Fig. S1a). Indeed, our data show that cells at time points immediately

57 following the first stain produce slightly more daughters than those at later time

58 points (linear model; $\mathrm{p}=0.03$ ). Taken together, these two sets of comparisons 
59 suggest that TrackScar does not significantly perturb the division rate of $S$. cerevisiae

60 cells.

\section{TrackScar provides a sensitive measure of differences in fecundity}

62 To determine the sensitivity of TrackScar, we tested whether this method

63 could detect the increased cell cycle time of daughter cells. S. cerevisiae reproduces

64 by asymmetrical division, and smaller daughter cells must grow longer until they

65 reach a critical size threshold before dividing for the first time (Hartwell and Unger

66 1977). Thus, daughter cells should have lower fecundity than mother cells that had

67 already divided at least once. Consistent with this expectation, daughter cells of

68 haploid strain S288C produced an average of 4.9 daughters in a six-hour period,

69 whereas mother cells produced an average of 5.4 daughters (Fig. S1b). This

70 difference is significant (Paired t-test; $n=3 ; p=0.030$ ). These results indicate that

71 TrackScar can accurately detect small differences in fecundity between individual

72 cells.

\section{Population Growth Rate and Mean Fecundity Are Well Correlated}

74 The different growth rates of sensitive and robust strains provided another

75 opportunity to test the accuracy of TrackScar. We used two-color TrackScar to

76 estimate fecundity at 30C and 35.5C for 8 sensitive and 13 robust strains. We

77 compared these estimates of average fecundity to the estimates of growth rate

78 obtained from growth curves. The average fecundity of cells measured using 
79 TrackScar and the maximum population growth rate measured by optical density at

$80 \quad 35.5 \mathrm{C}$ are well-correlated $\left(r^{2}=0.58\right.$; Fig. S2).

\section{Six Hours of Recovery Is Sufficient To Distinguish Live from Dead Cells}

82 Three-Color TrackScar relies on a sufficiently long recovery phase to

83 distinguish between live and dead cells. We tested whether six hours is sufficient to

84 distinguish live from dead cells by determining if YJM693 cells that did not divide

85 after six hours of recovery would subsequently form a colony on an agar plate.

86 We grew YJM693 to early-log phase at 35.5C as and stained the cells with

87 Ax488-WGA as described in the methods for the TrackScar protocol. After washing

88 off the stain, we spread the cells on a plate of YPD and randomly selected cells from

89 the patch of cells on the plate. Cells were immediately singled and subsequently

90 moved into a grid on the YPD plate. If a cell divided in between the time that the cell

91 was singled and when it was moved to the grid, both the mother cell and its progeny

92 were placed in the same space on the grid. The plate was incubated for $6 \mathrm{hr}$ at $30 \mathrm{C}$

93 and colonies were inspected for new daughter cells using a microscope. After 48hr,

94 the number of colonies formed by founder cells was recorded. Out of 24 cells that

95 did not divide after six hours of recovery at 30, only one of these these cells

96 subsequently divided in the next 48 hours. This indicates that six hours of recovery

97 in good conditions is adequate to distinguish live cells from dead cells. 
101 in the population would be approximately $1 / 2^{n}$ if age had no influence on fecundity

102 or mortality (the asymmetric division of yeast leads to a more complicated actual

103 distribution of ages that approximates $1 / 2^{n}$ when the doubling time of the

104 population is short (Lord and Wheals 1980)). Therefore, without specifically

105 enriching for old cells, it should be quite rare to observe cells that have budded

106 more than about ten times as they should make up less than 1 in 1,000 cells in the

107 population. However, as described above, heat stress can induce changes in age

108 related patterns of fecundity and mortality. One would predict therefore that some

109 stress related changes, particularly those that deplete young cells, could be

110 sufficient to alter population demography broadly.

111 We found that in the absence of heat stress, the distribution of ages in the

112 populations of all three strains approximately matched the $1 / 2^{n}$ expectation,

113 consistent with no influence of age. Furthermore, neither YJM693 nor S288C

114 showed significantly different age distributions at $30^{\circ} \mathrm{C}$ and $35.5^{\circ} \mathrm{C}$ (Kolmogorov-

115 Smirnov test, $p>0.3$ ). However, YJM996 had a significantly different distribution of

116 ages during growth at $35.5^{\circ} \mathrm{C}$ (Kolmogorov-Smirnov test, $\left.p=1.74 \times 10^{-7}\right)$. This

117 difference is due to a strong enrichment of cells older than five buds and a depletion

118 of cells between 2 and 4 buds (Fig. S6). Consequently, we frequently found cells with

119 more than 15 buds in YJM996 populations during growth at $35.5^{\circ} \mathrm{C}$. This is

120 consistent with the low fecundity and increased mortality we observed in young 
121 cells and indicates that heat stress can substantially alter the age distribution of a

122 population of cells by affecting their demographic rates.

\section{Supplementary Methods}

\section{TrackScar Staining}

125 All of our experiments were conducted using recently clonal cell lines in

126 early- to mid-log-phase growth, grown in YPD media (Sherman 2002), except for

127 experiments to visualize mitochondrial morphology, which where conducted in

128 synthetic media lacking uracil with $2 \%$ dextrose (SC-ura) media. To begin each

129 experiment, frozen stocks were streaked onto YPD or SC-ura plates to obtain single

130 colonies and incubated at $30^{\circ} \mathrm{C}$. After 24 to $48 \mathrm{hrs}$, single colonies were inoculated

131 into $2 \mathrm{ml}$ of YPD or SC-ura overnight at $30^{\circ} \mathrm{C}$.

132 To obtain log-phase cells, overnight cultures were diluted 1:100 and then in a

133 1:4 serial dilution in the columns of a 96 well plate. The final volume in each well of

134 the plate was $150 \mu \mathrm{l}$. The diluted cells were then placed in an incubator and shaken

135 at 500rpm at the desired temperature for at least $16 \mathrm{hr}$. Dilutions containing

136 putative early log-phase cultures were located by examining the optical density of

137 cells by eye and were then counted in a hemocytometer. A dilution whose

138 concentration was between $0.7 \times 10^{6}$ and $7 \times 10^{6}$ cells $/ \mathrm{ml}$ was chosen.

139 Log-phase cells were gently spun out of solution by centrifugation at 500xg

140 for 1.5 minutes. All centrifuge steps on live cells were done at this speed. Cells were

141 stained in $60 \mu \mathrm{l}$ of fresh media with $33 \mu \mathrm{g} / \mathrm{ml}$ wheat germ agglutinin (WGA) 
142 conjugated to Alexa488 (Invitrogen, W11261) at 30C for $15 \mathrm{~min}$ on a rotor. Cells

143 were washed once in $200 \mu \mathrm{l}$ of fresh media then diluted to $0.33 \times 10^{6}$ cells $/ \mathrm{ml}$ in one

144 column of a 96 well plate. Cells were then placed back in the incubator for $6 \mathrm{hr}$.

145 To fix the cells for subsequent imaging, cells were spun out of solution at

$1465,000 x g$ for 2 minutes. All subsequent centrifugation steps were done at this speed.

147 Cells were resuspended in $500 \mu$ l of sterile PBS. Cells were fixed by adding $100 \mu$ of

$14837 \%$ formaldehyde to the PBS and incubating at room temperature for at least 15

149 minutes. Cells were washed once in sterile PBS then resuspended in sterile PBS and

150 stored at $4^{\circ} \mathrm{C}$ in the dark until imaging. Cells were generally imaged less than a week

151 after collection, but the staining was stable for at least three months.

152 Immediately prior to imaging, cells were spun out of PBS and resuspended in

$15360 \mu \mathrm{l}$ of fresh media with WGA conjugated with tetramethylrhodamine (Invitrogen,

154 W849) for 15 minutes at $30^{\circ} \mathrm{C}$ on a rotor. Cells were washed $1 \mathrm{x}$ in $500 \mu \mathrm{l}$ of sterile

155 PBS, then sonicated in six short bursts using a Branson Sonifier 150 at setting 4 to

156 break up any clumps.

157 For experiments examining mitochondrial morphology, TrackScar staining

158 was performed as above, except the first WGA stain was WGA-TMR and the second

159 WGA stain six hours later was WGA-640CF (Biotium).

160 For the "Three-Color TrackScar" experiments used to determine if cells with

161 low fecundity during heat stress would divide subsequently after incubation at 30C,

162 cells were: 1) stained as above with WGA-Ax488 at the beginning of the experiment;

163 2) stained as above with WGA-640CF after 6 hr of incubation at either $35.5^{\circ} \mathrm{C}$ or 
$16440^{\circ} \mathrm{C} ; 3$ ) stored overnight at $4^{\circ} \mathrm{C}$ in YPD (for experimental convenience); 4)

165 incubated in YPD for 6hr at 30C on a rotor drum; 5) fixed with formaldehyde as

166 above and stored, and; 6) stained as above with WGA-TMR.

\section{Microscopy}

To count the stained bud scars for all experiments except for the time series

169 in Fig. S1 (see below), cells were imaged on a DeltaVision wide-field fluorescent

170 microscope using a 100x lens. Since cells that do not have the first stain (WGA-

171 Ax488) do not have any information about the number of daughters produced by

172 the cell, we randomly imaged cells that were stained with the first stain. To find cells

173 to image, we moved in transects along the slide and imaged each cell that had

174 staining in the Ax488 channel. The exception to this was when we sought out old

175 cells specifically in order to measure the fecundity or mortality of older cohorts. In

176 this case, we counted the number of buds that were visible in the Ax488 channel and

177 ignored young cells.

178 For each field of view containing a cell, ten- to twelve-micron deep z-stacks

179 with 0.2 to 0.4 micron slices were collected at every point with a 0.1 second

180 exposure with filter sets appropriate for each fluorophore being used in that

181 experiment. The depth of the z-stacks was chosen to ensure that both sides of every

182 cell were imaged. To visualize cell outlines, z-stacks of Nomarski bright-field images

183 were collected with a 0.025 second exposure. Either $768 \times 768$ or $1024 \times 1024$ images

184 were collected. Z-stacks were deconvolved using the default settings of the

185 DeltaVision software. The deconvolved z-stacks were cropped to remove 
186 deconvolution artifacts. Each channel was projected onto a single slice using a

187 maximum pixel intensity projection. At least 150 images (containing at least one

188 cell) for each sample were collected.

To generate images for display items in the figures, the brightness and

190 contrast of each cell and each channel was adjusted independently so that the first

191 stain would be visible. The gamma of the brightfield channel was sometimes

192 adjusted in order to remove saturated pixels that made display of the brightfield

193 image difficult.

\section{Image processing and data analysis}

195 The projected images of each z-stack were assembled into TIFF stacks using

196 a custom Python script and ImageMagick. Individual cells with the first TrackScar

197 stain (either Ax488 or TMR) were cropped from the wider images using custom

198 ImageJ macros. To count the bud scars, a false-color image of the individual cell was

199 displayed. To aid in the counting, we wrote ImageJ macros that allowed the rapid

200 toggling of different channels. Each bud scar in each channel was counted using the

201 following rules: 1) the birth scar is always counted as the first scar; 2) if a bud scar

202 has any staining of the previous stain that is visible at a normal brightness and

203 contrast level, then that bud scar is counted as being stained by that stain; 3) bud

204 scars that correspond to daughter cells still attached to the mother cell are not

205 counted unless the daughter cell has divided (has a bud scar besides its birth scar).

206 Mitochondrial morphology for Fig. S7 was scored by examining at least 100

207 random cells for each biological replicate in an Axio Imager and using a 100x lens. 
208 The field aperture was adjusted so that only one cell was visible at a time, and each

209 cell on a transect was scored. The presence of clumps of mitochondria and

210 mitochondrial threads was scored by examining cells using the appropriate filterset

211 for GFP. Mitochondrial morphology for Fig. S8 was scored by examining maximum

212 projections of Z-stacks created using a DeltaVision microscope as above for at least

213150 cells for each biological replicate. The presence of clumps of mitochondria and

214 mitochondrial threads was scored before examining the TrackScar staining for each

215 cell.

All statistical tests were performed using R (R Core Team 2015). To estimate

217 the division rate of haploid S288C cells, we fit a linear model to a plot of buds

218 produced as a function of time. To test for the effect of age on the average fecundity

219 of a cohort, we computed the average fecundity of the cohort of each strain for three

220 biological replicates and fit a linear model to those averages.

\section{Data collection and microscopy for time series}

222 To collect the time series in Fig. S1, we used the diploid strains YJM1529,

223 YJM1549, YJM1573, YJM195, YJM554, YJM555, YJM693, and S288C as well as

224 haploid S288C cells. TrackScar staining was done as above except the time between

225 the two stains was varied between one and six hours. To count the number of

226 daughters produced by cells in the time series in Fig. S1, the number of buds on at

227 least fifteen random cells stained by each stain were counted were counted using an

228 Axio Imager and a 100x lens in "real time" (without acquiring images). To see buds

229 on both sides of the cell, the plane of focus was moved up and down. 


\section{Growth curves}

231 To measure the maximum population growth rate of strains in the 100

232 genomes collection, 93 strains (see Table 1) were grown overnight in a 96 well plate

233 with $100 \mu \mathrm{l}$ of YPD per well at the desired temperature on a plate shaker at $280 \mathrm{rpm}$.

234 Cultures were mixed and diluted 1:5000 into a 96 well plate with $100 \mu$ l of YPD per

235 well. Growth curves were measured in a Tecan Sunrise plate reader. Measurements

236 were collected every $15 \mathrm{~min}$ for $48 \mathrm{hr}$ with 30 s of shaking at the highest setting

237 before each measurement. Four biological replicates were collected for the

238 temperatures $30^{\circ} \mathrm{C}$ and $35.5^{\circ} \mathrm{C}$ and at least two replicates were collected for all

239 other temperatures.

240 The maximum growth rate of a culture was calculated using non-parametric

241 smoothing using the "cellGrowth" package from Bioconductor. To identify strains

242 sensitive to heat stress, we looked for strains whose maximum population growth

243 rate at $35.5^{\circ} \mathrm{C}$ was $93 \%$ or lower than their growth rate at $30^{\circ} \mathrm{C}$. This cutoff was

244 chosen because S288C populations grew $94 \%$ as quickly at $35.5^{\circ} \mathrm{C}$ at $30^{\circ} \mathrm{C}$.

\section{Strain construction}

246 To generate the uracil auxotrophs CMY145 and CMY146 in the YJM693 and

247 YJM996 genetic background, respectively, the URA3 gene was disrupted by a

248 KanMX3 cassette. The plasmid M3927 (Voth, Wei Jiang, and Stillman 2003) (aka

249 PMB161) was digested using BamHI, then transformed into YJM693 and YJM996

250 using LiAc/PEG transformation (Gietz and Schiestl 2007). G418 resistant colonies 
251 were checked for the insertion of the cassette using colony PCR and strains with the

252 insertion of the cassette were sporulated. Tetrads were dissected and G418

253 resistant, uracil auxotrophs were identified by replica plating. To visualize

254 mitochondrial morphology, the plasmid p416-GPD-mito-roGFP1 (aka PMB273)

255 (McFaline-Figueroa et al. 2011)(a kind gift from Liza Pon) was transformed into

256 CMY145, CMY146, and PMY044 (a uracil auxotroph in the S288C background) to

257 yield CMY177, CMY178, and CMY182, respectively. 


\section{Supplementary References}

260

261 Gietz, R Daniel, and Robert H Schiestl. 2007. “High-Efficiency Yeast Transformation

262 Using the LiAc/SS Carrier DNA/PEG Method.." Nature Protocols 2 (1): 31-34.

263 doi:10.1038/nprot.2007.13.

264 Hartwell, L.H., and M W Unger. 1977. “Unequal Division in Saccharomyces

265 Cerevisiae and Its Implications for the Control of Cell Division..” The Journal of 266 Cell Biology 75 (2 Pt 1): 422-35.

267 Lord, P G, and A E Wheals. 1980. “Asymmetrical Division of Saccharomyces

268 Cerevisiae.." J. Bacteriol. 142 (3). American Society for Microbiology: 808-18.

269 Lord, P G, and A E Wheals. 1981. "Variability in Individual Cell Cycles of

270 Saccharomyces Cerevisiae ." Journal of Cell Science 50 (August): 361.

271 McFaline-Figueroa, José Ricardo, Jason Vevea, Theresa C Swayne, Chun Zhou,

272 Christopher Liu, Galen Leung, Istvan R Boldogh, and Liza A Pon. 2011.

273 "Mitochondrial Quality Control During Inheritance Is Associated with Lifespan

274 and Mother-Daughter Age Asymmetry in Budding Yeast..” Aging Cell 10 (5).

275 Blackwell Publishing Ltd: 885-95. doi:10.1111/j.1474-9726.2011.00731.x.

276 Sherman, Fred. 2002. "Getting Started with Yeast.." Methods in Enzymology 350: 3$277 \quad 41$.

278 Voth, Warren P, Yi Wei Jiang, and David J Stillman. 2003. “New 'Marker Swap’

279 Plasmids for Converting Selectable Markers on Budding Yeast Gene Disruptions

280 and Plasmids.” Yeast 20 (11). John Wiley \& Sons, Ltd.: 985-93.

281 doi:10.1002/yea.1018. 


\section{Supplementary Figures}
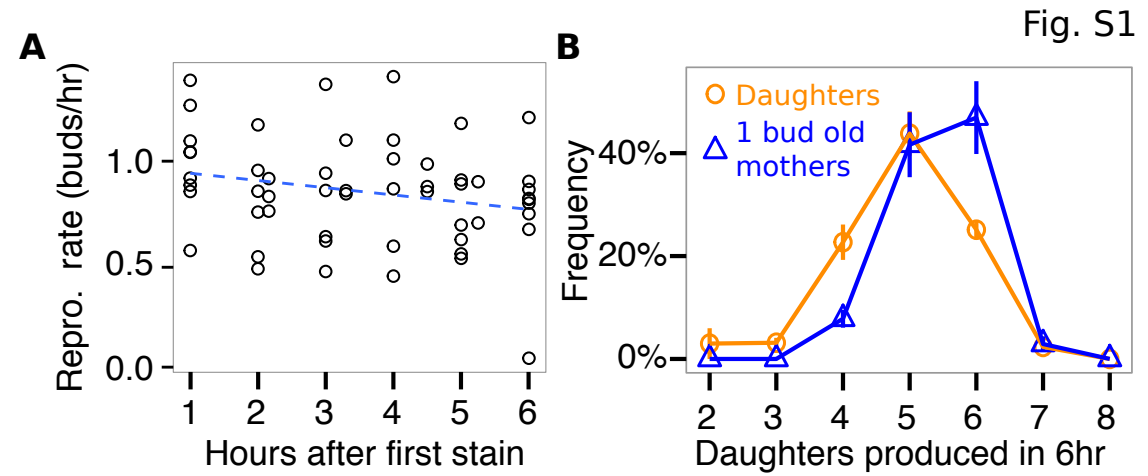

284 Figure S1. TrackScar is sensitive and minimally affects cellular physiology. A) The

285 average number of daughters produced in a one hour interval by cells in nine

286 genetically diverse yeast strains is shown as a function of time since the first WGA

287 stain. A linear regression line is plotted as a blue dashed line. B) Histograms of the

288 number of daughters produced by daughter cells (orange circles) and mother cells

289 that had divided once (blue triangles) in a 6hr interval for are shown. Error bars

290 show the standard error of the mean for three biological replicates. 
Fig. S2

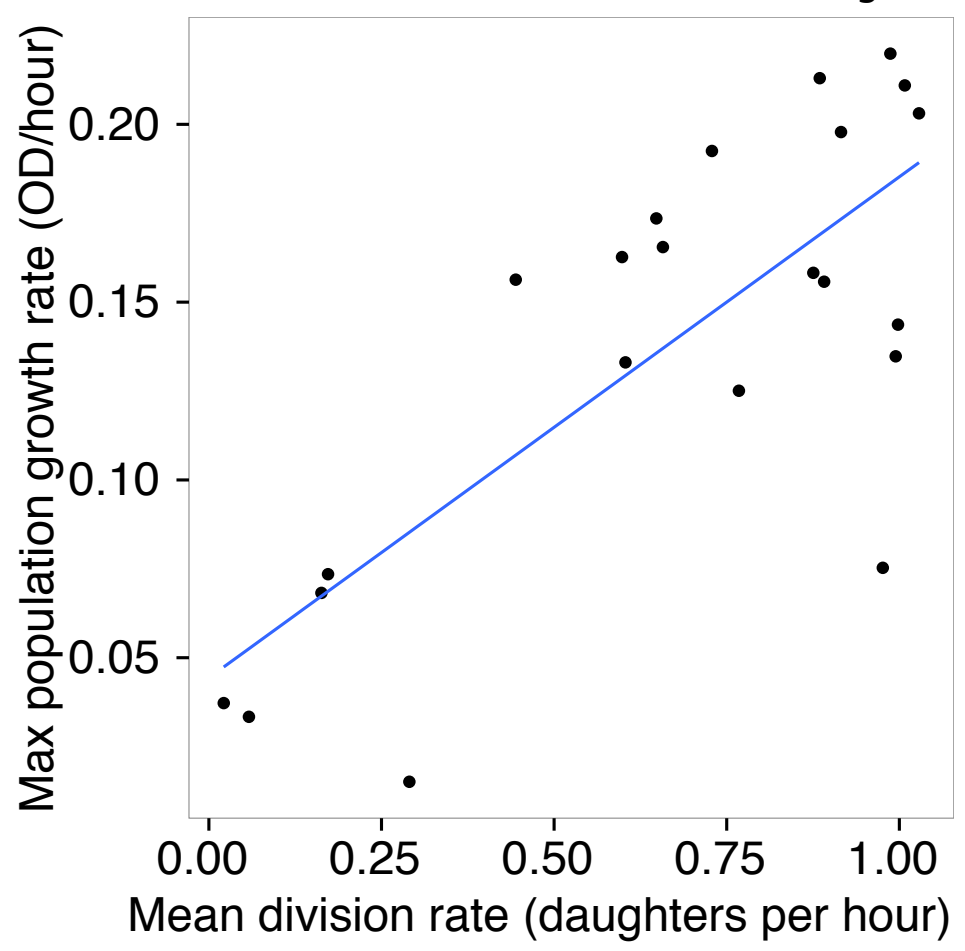

292 Figure S2. Estimates of the growth rate of a population made using TrackScar and

293 growth curves are well-correlated. A scatter plot of the max growth of a population

294 inferred by measuring its rate of increase in a spectrophotometer and the average

295 fecundity of a population measured using TrackScar is shown for a diverse panel of

296 yeast strains grown at 35.5C. A linear regression line is shown in blue. 


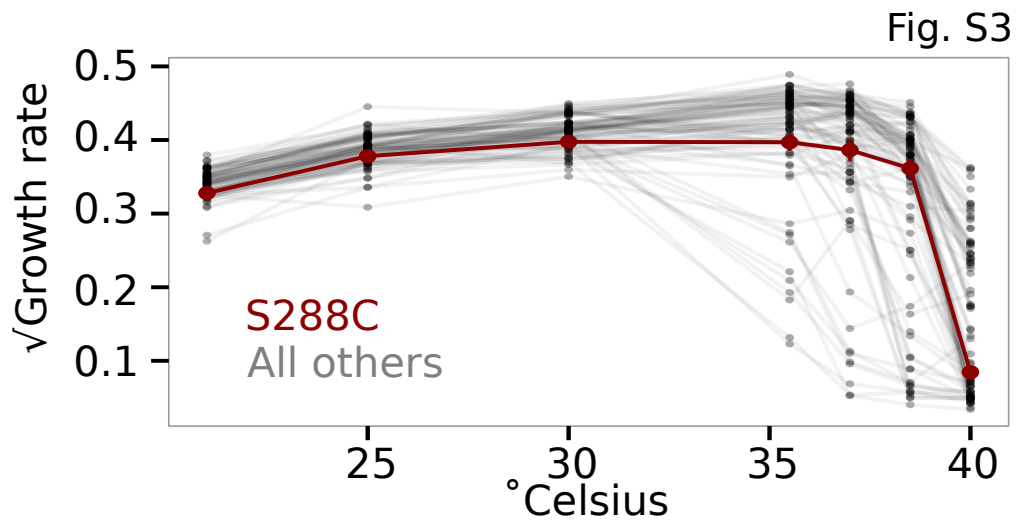

300 Figure S3. There is extensive genetic diversity in thermotolerance among

301 environmental yeast isolates. The square-root of the maximum growth of each of 93

302 yeast strains is shown as a function of temperature. The strain S288C is shown in

303 red. 


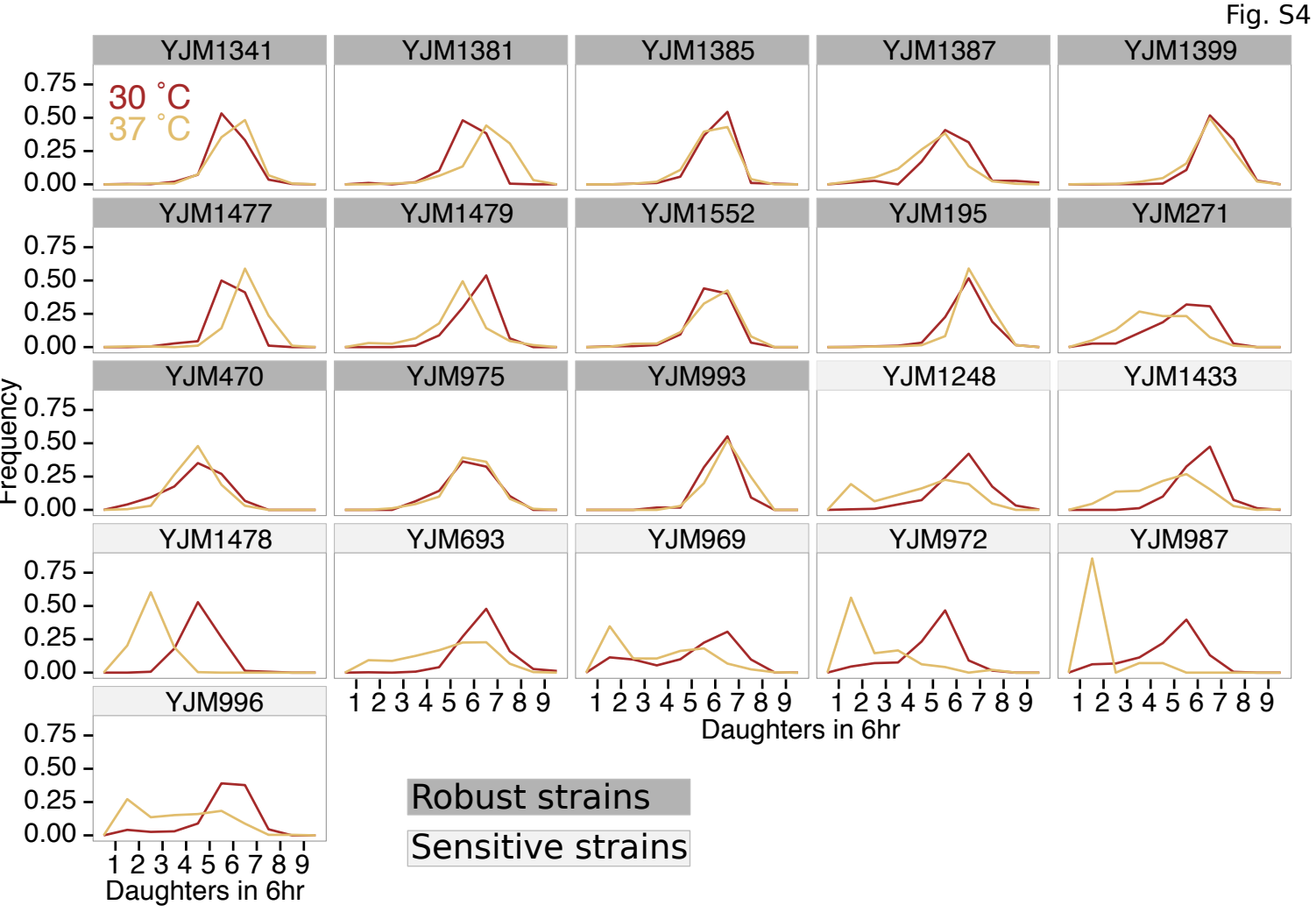

307 Figure S4. Sensitive strains frequently show sub-populations of cells with very low

308 fecundity. Plots showing histograms of the number of daughters produced in $6 \mathrm{hr}$ at

309 30C (brown) and 35.5C (gold) are shown for all strains that were examined using

310 TrackScar. Strains that are robust to heat stress have their facet labels colored in

311 dark grey. 
Fig. S5

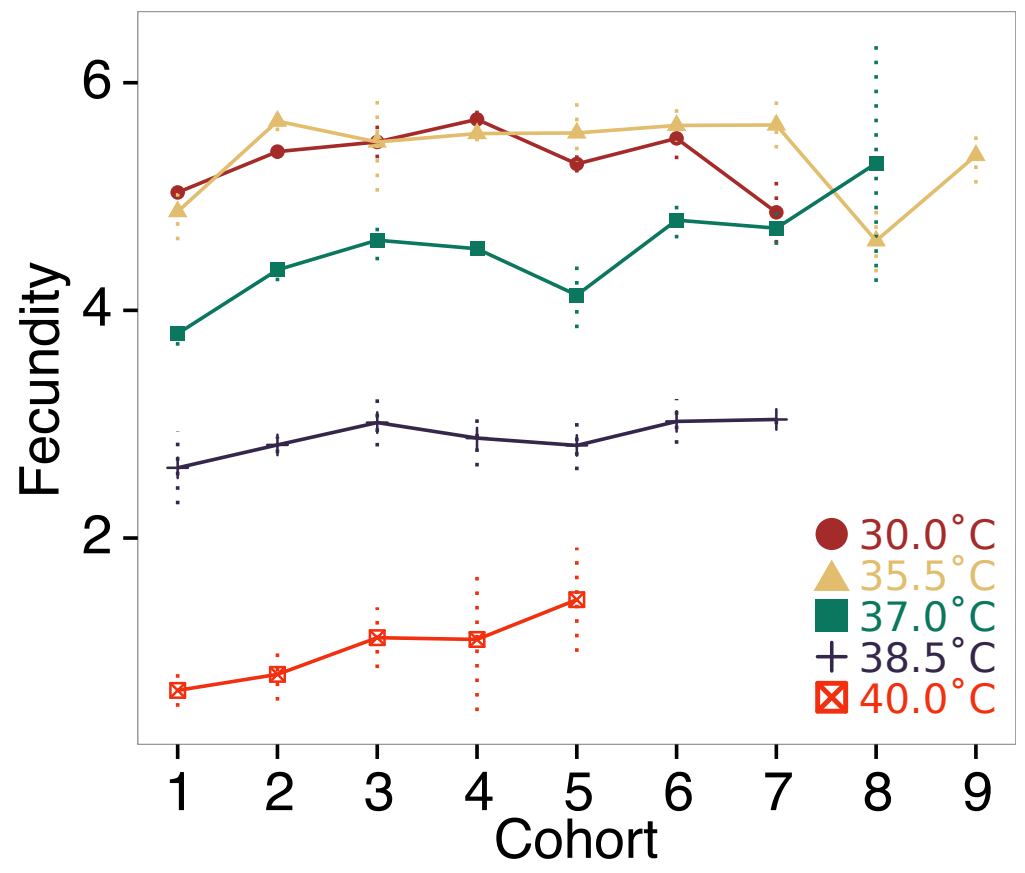

313 Figure S5. The fecundity of S288C cells is not age structured at any temperature.

314 The average fecundity of cohorts of S288C cells during 6hr during growth at 30C

315 (brown circles), 35.5C (gold triangles), 37C (green squares), 38.5C (black crosses),

316 and 40C (red crossed boxes). Error bars are the standard error of the mean for at

317 least two biological replicates. Only cohorts with at least three cells in each of the

318 biological replicates are shown. 


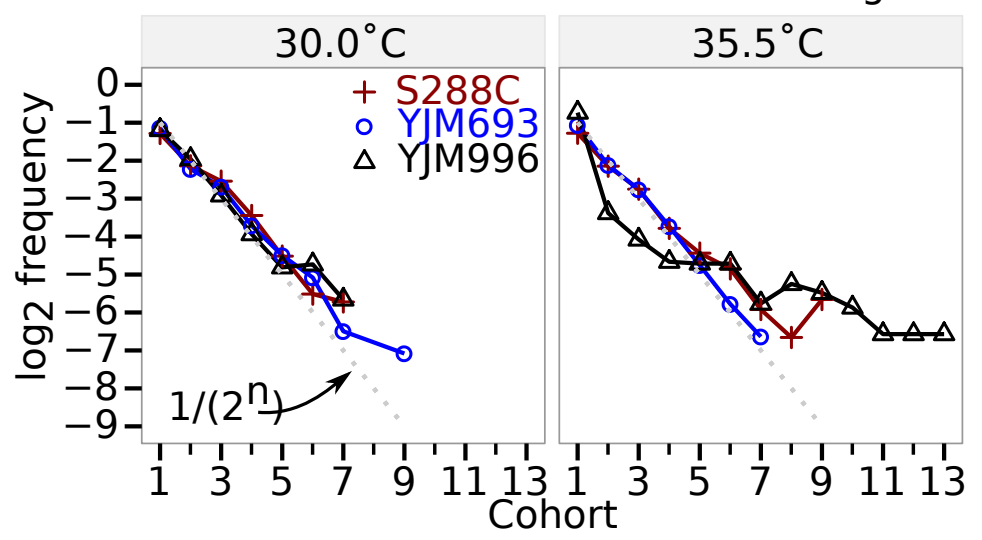

321 Figure S6. Heat stress can alter the distribution of ages in a population. The

322 frequency of cells in a cohort in a sample of randomly chosen cells at 30C and 35.5C

323 is shown for the strains S288C (red crosses), YJM693 (blue circles), and YJM996

324 (black triangles) is shown. The grey dotted line shows $1 /\left(2^{n}\right)$. Each point represents

325 the pooled observations across at least three biological replicates. Only cohorts with

326 at least five total cells are shown. 


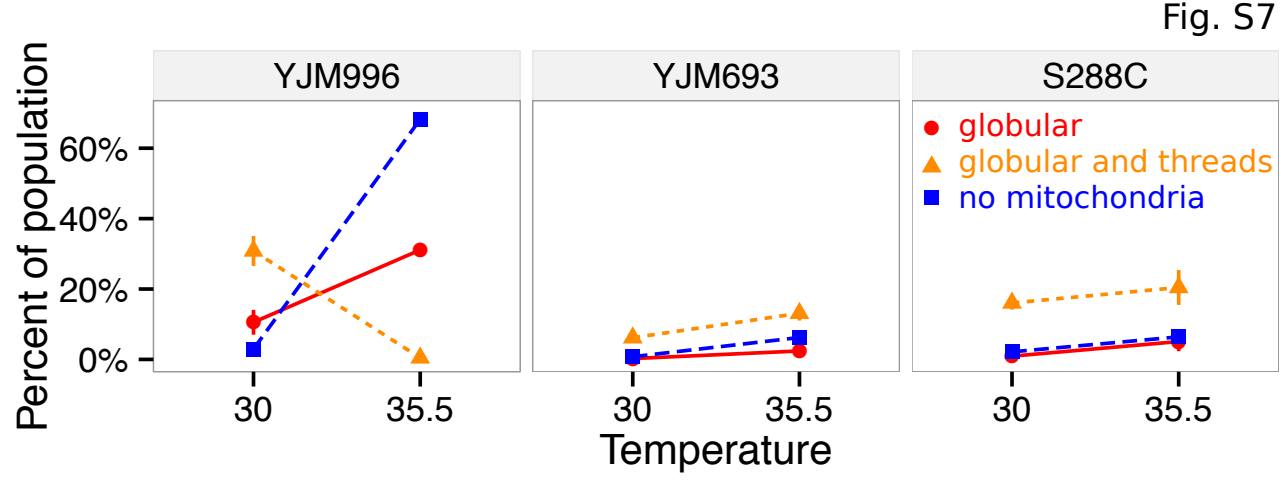

329 Figure S7. Heat stress changes the frequency of cells with abnormal mitochondrial 330 morphology in the strain YJM996. The percentage of the population with abnormal 331 mitochondria at $30^{\circ} \mathrm{C}$ and $35.5^{\circ} \mathrm{C}$ is shown for the strains CMY177 (YJM693 genetic 332 background), CMY178 (YJM996 genetic background), and CMY182 (S288C genetic

333 background). Mitochondria were grouped into the classes: globular (red circles),

334 globular with some threads (orange triangles), and no mitochondria (blue squares).

335 Error bars show the standard error of the mean for three biological replicates of at 336 least 100 cells.

\section{Supplementary Tables}

338 Supp. Table 1 Strains used in this study. See attached file "TableS1 - strains.xlsx" 